\title{
Liver Transplantation in Children and Impact of Intraoperative Goal Directed Therapies on Postoperative Outcome
}

\author{
Claudine Kumba* \\ Department of Pediatric Anesthesia and Critical Care, Hôpital Universitaire Necker, France. \\ ORCID ID: https://orcid.org/0000-0002-9748-5141
}

\begin{abstract}
Background: Liver transplantation is a hemorrhagic surgery with high postoperative complication rates in terms of organ dysfunction and infections. We conducted a retrospective observational monocentric study that had the objectives of determining predictors of postoperative outcome in children. In the initial cohort, 19 children underwent liver transplantation. A description of the postoperative outcomes of these patients was undertaken.
\end{abstract}

Objective: To describe intraoperative and postoperative outcomes in children undergoing liver transplantation and to propose intraoperative management implementation measures for postoperative outcome improvement.

Methods: Secondary analysis of liver transplanted patients included in the initial study.

Results: There were 19 patients who underwent liver transplantation with a median age of 31[2-154] months. Five patients had re-operations (26.3\%). Fourteen (73.7\%) had intraoperative and/or postoperative complications. Five (26.3\%) had intraoperative hemorrhagic shock. One (5.3\%) had postoperative respiratory failure, one (5.3\%) had postoperative multiple organ failure, two $(10.5 \%)$ had postoperative neurologic failure and five $(26.3 \%)$ had cardio-circulatory failure. One (5.3\%) had postoperative pulmonary sepsis, five (26.3\%) had postoperative abdominal sepsis, and six (31.6\%) had postoperative septicemia. Intra-operative transfusion rate was 100\%. In-hospital mortality rate was 10.5\% (two patients).

Conclusion: There were multiple postoperative adverse outcome predictors. Optimal intraoperative patient management with transfusion guided by point-of-care viscoelastic devices and goal-directed fluid and hemodynamic therapy with validated parameters and tools in children is a possible pathway to upgrade postoperative evolution in pediatric liver transplantation.

Keywords: Liver transplantation, Children, Transfusion, Outcome, Patient blood management, Goal directed therapies.

\section{Introduction}

Liver transplantation is one of the most hemorrhagic surgical interventions. Postoperative complication rates in terms of organ dysfunction and infections are high due to the major characteristic of the surgery and to the critical general status of patients requiring urgent transplantation. Intraoperative blood management and intraoperative fluid and hemodynamic monitoring are necessary for optimal postoperative outcomes. In our hospital, liver transplantation is among interventions that are performed most of the time on an emergency basis depending on the availability of transplant grafts and patient waiting lists. We conducted a retrospective observational monocentric study in our hospital, which had the
Quick Response Code:

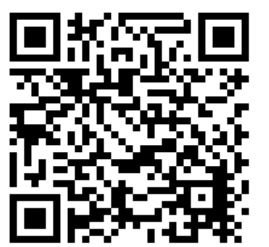

*Corresponding author: Claudine Kumba, Department of Pediatric Anesthesia and Critical Care, Hôpital Universitaire Necker Enfants Malades, Assistance Publique Hôpitaux de Paris, Université de Paris, 146 Rue de Sèvres, 75015 Paris, France

Received: 30 October, 2021

Published: 28 January, 2022

Citation: Kumba C. Liver Transplantation in Children and Impact of Intraoperative Goal Directed Therapies on Postoperative Outcome. SOJ Pedia Clin Neonato. 2022;2(1):1-7. DOI: 10.53902/SOJPCN.2022.02.000513 
objectives of determining predictors of postoperative outcome in pediatric surgical patients in neurosurgery, abdominal surgery and orthopedics. ${ }^{1}$ In this study of 594 patients, predictors of postoperative outcome were transfusion, American Society of Anesthesiologists score (ASA), emergency surgery, surgery and age. In this initial cohort, 19 children underwent liver transplantation. A secondary analysis was undertaken to describe intraoperative and postoperative outcomes in these liver transplanted patients. The aim of this study was to describe intraoperative and postoperative outcomes in these patients and to suggest implementation measures for optimal intraoperative management with the aim of improving postoperative evolution in surgical pediatric patients.

\section{Methods and Materials}

A secondary analysis of liver transplantation patients was included in the initial retrospective study. ${ }^{1}$ The study was declared to the National Commission for Computer Science and Liberties (CNIL) under registration number 2028257 v0 on 21 February 2017 and approved by the Ethics Committee of Necker under registration number 2017-CK-5-R1 on 21 March 2017. The inclusion criteria were patients under 18 years old who underwent hepatic transplantation and were included in the initial retrospective study. The exclusion criteria were patients aged more than 18 years old and patients who did not undergo hepatic transplantation included in the initial study. Patients were included retrospectively from 1 January 2014 to 17 May 2017. Statistics were analyzed with XLSTAT 2020.4.1. Software. Continuous variables were expressed as medians with ranges or means with standard deviations. Category variables were described in proportions.

In our hospital, liver transplantation was performed according to an established protocol as described hereafter. Before surgery, all patients underwent transthoracic echocardiography, hemoglobin levels, complete blood cell count, blood coagulation analysis, plasma electrolytes, complete liver function tests, blood group, and available blood products corresponding to 2-4 times the whole blood volume representing $40 \%$ of packed red blood cell units, $60 \%$ of fresh frozen plasma units and $0.2 \mathrm{U} / \mathrm{kg}$ of concentrated platelet units. Induction of anesthesia was performed with sevoflurane in an air-oxygen mixture. Induction of anesthesia was performed intravenously if inhalational anesthesia was contraindicated. Three large-bored peripheral intravenous lines of $>20 \mathrm{G}$ were inserted. Airway was secured with orotracheal intubation. Maintenance of anesthesia was performed with sevoflurane in an air-oxygen mixture and with a continuous intravenous infusion of sufentanil, ketamine and atracurium. All patients were monitored with a bispectral index, cerebral and renal oxygen saturation (NIRS, near infrared spectroscopy), central core temperature probe, and indwelling bladder catheter; one or two central venous lines were inserted if peripheral intravenous catheters were not sufficient, and a radial arterial catheter was inserted. Depending on patient cardiac status, hemodynamics was monitored with Flo-trac/Vigileo if the patient's weight was over $25 \mathrm{~kg}$ or with an esophageal Cardio-Q Doppler probe.

Fluid therapy was managed with crystalloids containing 1 liter of $2.5 \%$ glucose, $2 \mathrm{~g}$ sodium chloride, and $2 \mathrm{~g}$ calcium chloride at a basal infusion rate of $5-10 \mathrm{ml} / \mathrm{kg} / \mathrm{h}$ with supplemental fluid therapy at an infusion rate of $5-30 \mathrm{ml} / \mathrm{kg} / \mathrm{h}$ with $0.9 \%$ sodium chloride ( $\mathrm{NaCl} 0.9 \%$ ), albumin 5\%, fresh frozen plasma, packed red blood cells and platelet units. Fluid therapy was adjusted according to central venous pressure, pulse pressure variation, hemoglobin levels, platelet levels and bleeding. The objectives were to maintain hemoglobin levels above $10 \mathrm{~g} / \mathrm{dL}$, platelet levels $>50000 / \mu \mathrm{L}$, base excess $8-10 \mathrm{mmol} / \mathrm{L}$, plasma ionized calcium levels $>1 \mathrm{mmol} / \mathrm{L}$, glycemia between $5.5-11 \mathrm{mmol} / \mathrm{L}$, diuresis $>0.5-1 \mathrm{ml} / \mathrm{kg} / \mathrm{h}$, prothrombin time $>30 \%$, fibrinogen levels $>2 \mathrm{~g} / \mathrm{L}$, mean arterial pressure $>55 \mathrm{mmHg}$, central venous pressure between $4-10 \mathrm{mmHg}$, pulse pressure variation between $12-15 \%, \mathrm{pH}>7$ and normothermia. In case of uncontrolled bleeding, concentrated prothrombin complex (confidex@) was administered after discussion with the concerned team in charge. Recombinant factor VII (Novoseven $®$ ) was considered a rescue solution in cases of refractory bleeding after discussion with the team in charge because of the risk of arterial and venous thrombosis.

All patients received antibiotic prophylaxis with intravenous piperacillin/tazobactam or imipenem and vancomycin according to patient microbiologic status. Tranexamic acid was administered as an intravenous bolus of $25 \mathrm{mg} / \mathrm{kg}$ followed by an intravenous infusion of $2 \mathrm{mg} / \mathrm{kg} / \mathrm{h}$ up to 15 minutes before the end of arterial clamping. Tranexamic acid was not systematically administered in patients weighing less than $10 \mathrm{~kg}$ because of the risk of arterial and venous thrombosis and could be considered case by case after discussion with the team in charge of the patient. Fibrinogen was administered if plasmatic levels were lower than $2 \mathrm{~g} / \mathrm{L}$. Vasoactive drugs with norepinephrine or epinephrine were administered if necessary. Immunosuppressive therapy was administered with $10 \mathrm{mg}$ intravenous basiliximab (Simulect $\AA$ ) if the patient's weight was less than $35 \mathrm{~kg}$ or $20 \mathrm{mg}$ if the weight was more than $35 \mathrm{~kg}$ after hepatic clamping. Patients were transferred postoperatively sedated and intubated to the pediatric intensive care unit.

\section{Results}

Table 1 illustrates general characteristics. There were 19 patients who underwent liver transplantation with a median age of 31[2-154] months, median weight of 11.5 [4.5-39] kilograms, three patients (15.8\%) were ASA III and 16 (84.2\%) were ASA IV. All were operated on an emergency basis. There were five patients who had reoperations (26.3\%). Fourteen (73.7\%) had intraoperative and/ or postoperative complications. Five $(26.3 \%)$ had intraoperative 
hemorrhagic shock. One $(5.3 \%)$ had postoperative respiratory failure, one $(5.3 \%)$ had postoperative multiple organ failure, two $(10.5 \%)$ had postoperative neurologic failure and five $(26.3 \%)$ had cardio-circulatory failure. One $(5.3 \%)$ had postoperative pulmonary sepsis, five (26.3\%) had postoperative abdominal sepsis and six $(31.6 \%)$ had postoperative septicemia. All patients were transfused intra-operatively. The median packed red blood cell volume was 2[1-12] units, the median fresh frozen plasma volume was $5[0$ 16] units and the median concentrated platelet volume was 0 [0-6] units. The mean preoperative hemoglobin level was $9.8 \pm 1 \mathrm{~g} / \mathrm{dL}$, and the mean postoperative hemoglobin level was $10.6 \pm 1.5 \mathrm{~g} / \mathrm{dL}$. The median intraoperative crystalloid volume was $1168[130-7000] \mathrm{ml}$, and the median intraoperative colloid volume was $450[0-1700] \mathrm{ml}$.
The median length of intensive care unit stay (LOSICU) was 27[274] days. The median length of hospital stay (LOS) was 34[14-116] days, the median total length of hospital stay (TLOS=LOSICU+LOS) was 66[16-187] days, and the median total length of mechanical ventilation (LMV) was 4[0-39] days. The in-hospital mortality rate was $10.5 \%$ (in two patients) (Table 2). Patients with fatal outcomes had hepatic failure as a comorbidity and had intraoperative hemorrhagic shock. One patient had postoperative multiple organ failure with septicemia, and the second patient had postoperative cardio-circulatory failure with abdominal sepsis. Table 3 illustrates co-morbidities. The majority of the patients $(17,89.5 \%)$ had hepatic failure, one patient (5.3\%) had congenital heart disease and one $(5.3 \%)$ had metabolic disease.

Table 1: General characteristics of liver transplantation patients.

\begin{tabular}{|c|c|}
\hline Characteristic & $\mathrm{N}=19$ \\
\hline Median age [range] in months & $31[2-154]$ \\
\hline Median weight [range] in kilograms & $11.5[4.5-39]$ \\
\hline ASA III n (\%) & $3(15.8)$ \\
\hline ASA IV n (\%) & $16(84.2)$ \\
\hline Emergency surgery n (\%) & $19(100)$ \\
\hline Re-operation n (\%) & $5(26.3)$ \\
\hline Patients with intra-operative and or postoperative complications (organ failure or sepsis) n (\%) & $14(73.7)$ \\
\hline Intra-operative hemorrhagic shock n (\%) & $5(26.3)$ \\
\hline Postoperative cardio-circulatory failure n (\%) & $5(26.3)$ \\
\hline Postoperative miscellaneous n (\%) & $1(5.3)$ \\
\hline Postoperative multiple organ failure n (\%) & $1(5.3)$ \\
\hline Postoperative respiratory failure $\mathrm{n}(\%)$ & $1(5.3)$ \\
\hline Postoperative neurologic failure n (\%) & $2(10.5)$ \\
\hline Postoperative abdominal sepsis n (\%) & $5(26.3)$ \\
\hline Postoperative septicemia n (\%) & $6(31.6)$ \\
\hline Postoperative pulmonary sepsis n (\%) & $1(5.3)$ \\
\hline In-hospital mortality n (\%) & $2(10.5)$ \\
\hline Transfusion n (\%) & $19(100)$ \\
\hline Median packed red blood cell volume in units [range] & $2[1-12]$ \\
\hline Median fresh frozen plasma volume in units [range] & $5[0-16]$ \\
\hline Median concentrated platelet units [range] & $0[0-6]$ \\
\hline Mean preoperative hemoglobin levels \pm standard deviation in $\mathrm{g} / \mathrm{dL}$ & $9.8 \pm 1$ \\
\hline Mean postoperative hemoglobin levels \pm standard deviation in $\mathrm{g} / \mathrm{dL}$ & $10.6 \pm 1.5$ \\
\hline Median crystalloid volume in ml [range] & $1168[130-7000]$ \\
\hline Median colloid volume in ml [range] & $450[0-1700]$ \\
\hline Median length of intensive care unit stay in days [range] & $27[2-74]$ \\
\hline Median length of hospital stay in days [range] & $34[14-116]$ \\
\hline Median total length of hospital stay in days [range] & $66[16-187]$ \\
\hline Median total length of mechanical ventilation in days [range] & $4[0-39]$ \\
\hline
\end{tabular}


Table 2: Patients with fatal outcome.

\begin{tabular}{|l|l|l|l|l|l|l|l|}
\hline Surgery & $\begin{array}{l}\text { Age in } \\
\text { months }\end{array}$ & $\begin{array}{l}\text { ASA } \\
\text { score }\end{array}$ & $\begin{array}{l}\text { Co-morbid- } \\
\text { ities }\end{array}$ & $\begin{array}{l}\text { Intra-operative } \\
\text { complications }\end{array}$ & $\begin{array}{l}\text { Postoperative } \\
\text { outcome }\end{array}$ & $\begin{array}{l}\text { Delay of in-hos- } \\
\text { pital mortality } \\
\text { in days }\end{array}$ & $\begin{array}{l}\text { Transfu- } \\
\text { Emergency } \\
\text { sion }\end{array}$ \\
\hline $\begin{array}{l}\text { Liver Trans- } \\
\text { plantation }\end{array}$ & 5 & 4 & $\begin{array}{l}\text { Hepatic } \\
\text { failure }\end{array}$ & $\begin{array}{l}\text { Hemorrhagic } \\
\text { shock }\end{array}$ & $\begin{array}{l}\text { Multiple Organ } \\
\text { Failure and Septi- } \\
\text { cemia }\end{array}$ & 148 \\
\hline $\begin{array}{l}\text { Liver Trans- } \\
\text { plantation }\end{array}$ & 62 & 4 & $\begin{array}{l}\text { Hepatic } \\
\text { Failure }\end{array}$ & $\begin{array}{l}\text { Hemorrhagic } \\
\text { shock }\end{array}$ & $\begin{array}{l}\text { Cardio-circulatory } \\
\text { failure and Ab- } \\
\text { dominal Sepsis }\end{array}$ & 86 \\
\hline
\end{tabular}

Table 3: Co-morbidities.

\begin{tabular}{|l|l|}
\hline Co-morbidity & Number of patients (\%) \\
\hline Congenital heart disease & $1(5.3)$ \\
\hline Metabolic disease & $1(5.3)$ \\
\hline Hepatic failure & $17(89.5)$ \\
\hline
\end{tabular}

\section{Discussion}

Fourteen patients among 19 (73.7\%) had intraoperative or postoperative complications. Intraoperative hemorrhagic shock was observed in five $(26.3 \%)$ patients. Liver transplantation is one of the most hemorrhagic surgical interventions. Preoperative predictors of intraoperative bleeding in hepatic transplantation have been described previously. ${ }^{2}$ All patients were transfused intraoperatively with packed red blood cells and/or fresh frozen plasma and/ or platelet units. Transfusion with all sorts of blood products, which is a necessary therapeutic in hemorrhagic surgery, has been correlated with adverse postoperative outcomes in terms of organ dysfunction, length of hospital and intensive care unit stays and length of mechanical ventilation in critically ill patients. ${ }^{1-5}$ In children, patient blood management programs with goal-directed transfusion guided with point-of-care viscoelastic devices have been shown to reduce intraoperative fresh frozen plasma and length of hospital stay in hemorrhagic interventions such as liver transplantation. ${ }^{6}$ In our hospital, we have not yet integrated point-of-care viscoelastic devices to guide transfusion in liver transplantation surgery. With existing evidence, it is necessary to integrate these devices into patient blood management programs in this surgical setting. As an improvement implementation measure, a protocol with a pointof-care device, namely, rotational thromboelastometry (ROTEM), should be integrated into patient blood management in liver transplantation, as illustrated in Figures 1-3. Pediatric values of ROTEM have been described previously with ROTEM delta version. ${ }^{7}$

Hemodynamic monitoring depends on the patient's general status and surgery. Liver transplantation is a major surgery, and hemodynamic monitoring with validated tools and parameters in children is necessary for intraoperative optimization and should be applied systematically in all patients undergoing major surgery. Pulse pressure variation and central venous pressure are not optimal parameters to guide fluid therapy in children. ${ }^{8}$ In pediatrics, fluid therapy management in terms of fluid responsiveness is best as- sessed with aortic peak blood flow velocity variation, which can be measured with echocardiography or an esophageal Doppler probe (Cardio-Q). ${ }^{8,9}$ If the FloTrac/Vigileo system is used for fluid and vasoactive therapy, stroke volume variation, which is determined with arterial pressure waveform analysis, can be used to assess fluid responsiveness; however, this device has not yet been validated for fluid therapy in pediatric surgical settings. ${ }^{10,11}$ One study that included 23 patients aged 12 to 65 years in posterior spine arthrodesis surgery showed that goal-directed therapy guided by FloTrac/ Vigileo stroke volume variation decreased intraoperative bleeding and blood product requirements, postoperative pulmonary complications, intensive care unit stay and postoperative gastrointestinal complications. ${ }^{12}$ Thus, pediatric studies in surgical settings with FloTrac/Vigileo goal-directed fluid and hemodynamic therapy are necessary to determine the impact of this device on postoperative outcome. Monitoring hemodynamics with validated parameters in children is mandatory for optimal intraoperative management in major pediatric surgery, which has the objectives of reducing postoperative organ dysfunction. A systematic review and meta-analysis in children demonstrated that nonoptimal intraoperative hemodynamic biomarkers such lactate levels, central venous mixed oxygen saturation (SVO2), and regional cerebral and renal oxygen saturation assessed by near-infrared spectroscopy (NIRS) were correlated with adverse postoperative outcomes in pediatric surgical settings in terms of morbidity, mortality and length of hospital stay. ${ }^{13}$ Monitoring and optimizing these biomarkers should be part of intraoperative patient management in critically ill patients and major surgery. Postoperative outcomes depend on multiple predictors. ${ }^{1-5}$ In this study, the rates of postoperative cardio-circulatory, neurologic, multiple organ and respiratory failure were $26.3,10.5$, 5.3 and $5.3 \%$, respectively. The rates of postoperative septicemia and abdominal and pulmonary sepsis were 31.6, 26.3 and 5.4\%, respectively. In-hospital mortality rate was $10.5 \%$. Integrating intraoperative goal-directed therapy with validated tools in children is necessary to reduce postoperative adverse outcomes. However, studies evidencing the impact of intraoperative goal-directed fluid and hemodynamic therapy on postoperative outcomes in pediatrics are still lacking; therefore, developing research in this field will contribute to clarifying these issues in domains where many questions are still unanswered..$^{14-18}$ 


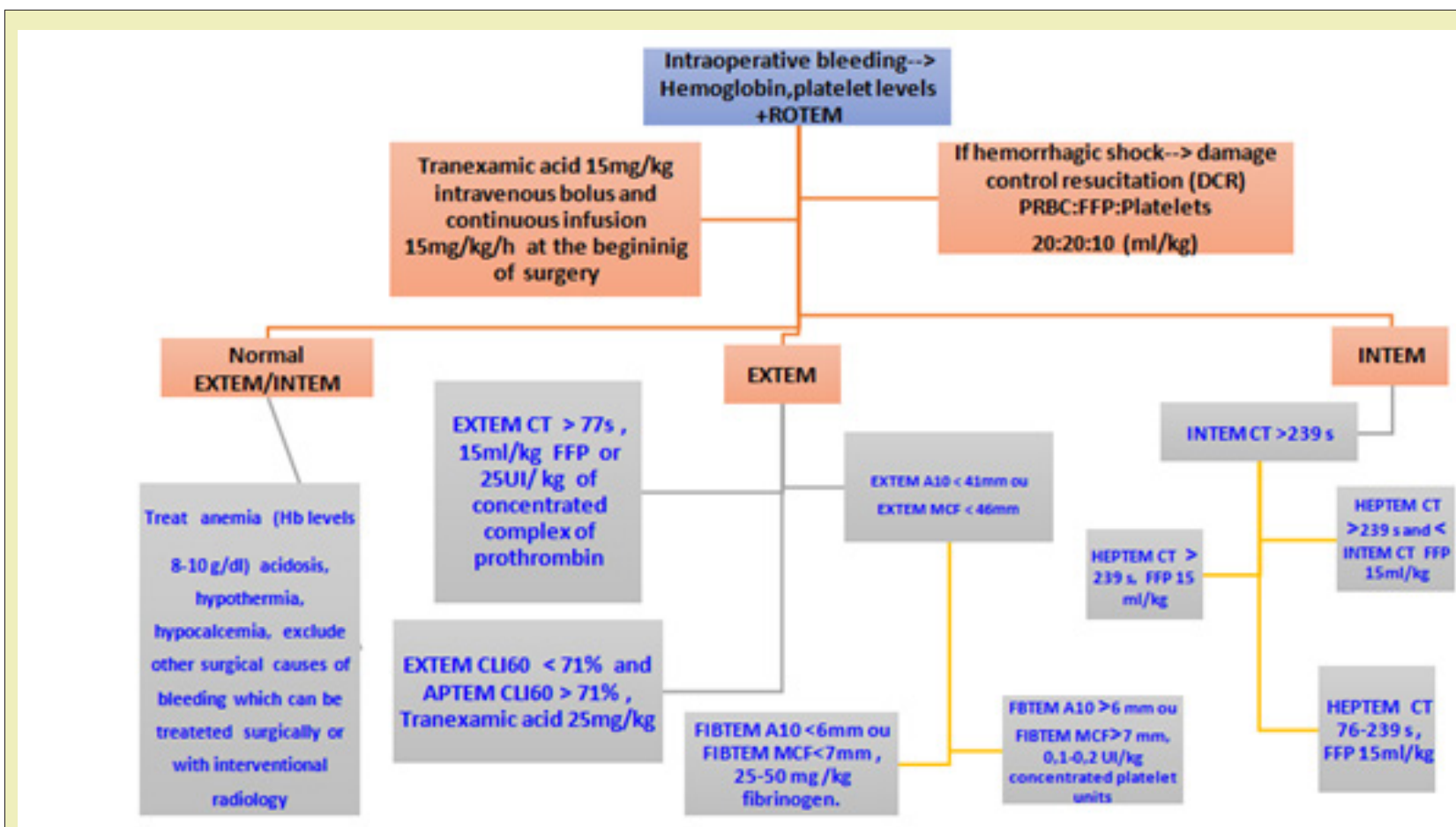

Figure 1: ROTEM Algorithm in children between 0-24 months.

$\mathrm{CT}=$ coagulation time in seconds, $\mathrm{A} 10=$ clot firmness at 10 minutes, $\mathrm{MCF}=$ maximum clot firmness, $\mathrm{CLI} 60=$ lysis index in $\% 60$ minutes after $\mathrm{CT}$, ML= maximum lysis in \%, FFP=fresh frozen plasma, $\mathrm{PRBC}=$ packed red blood cells, $\mathrm{Hb}=$ hemoglobin

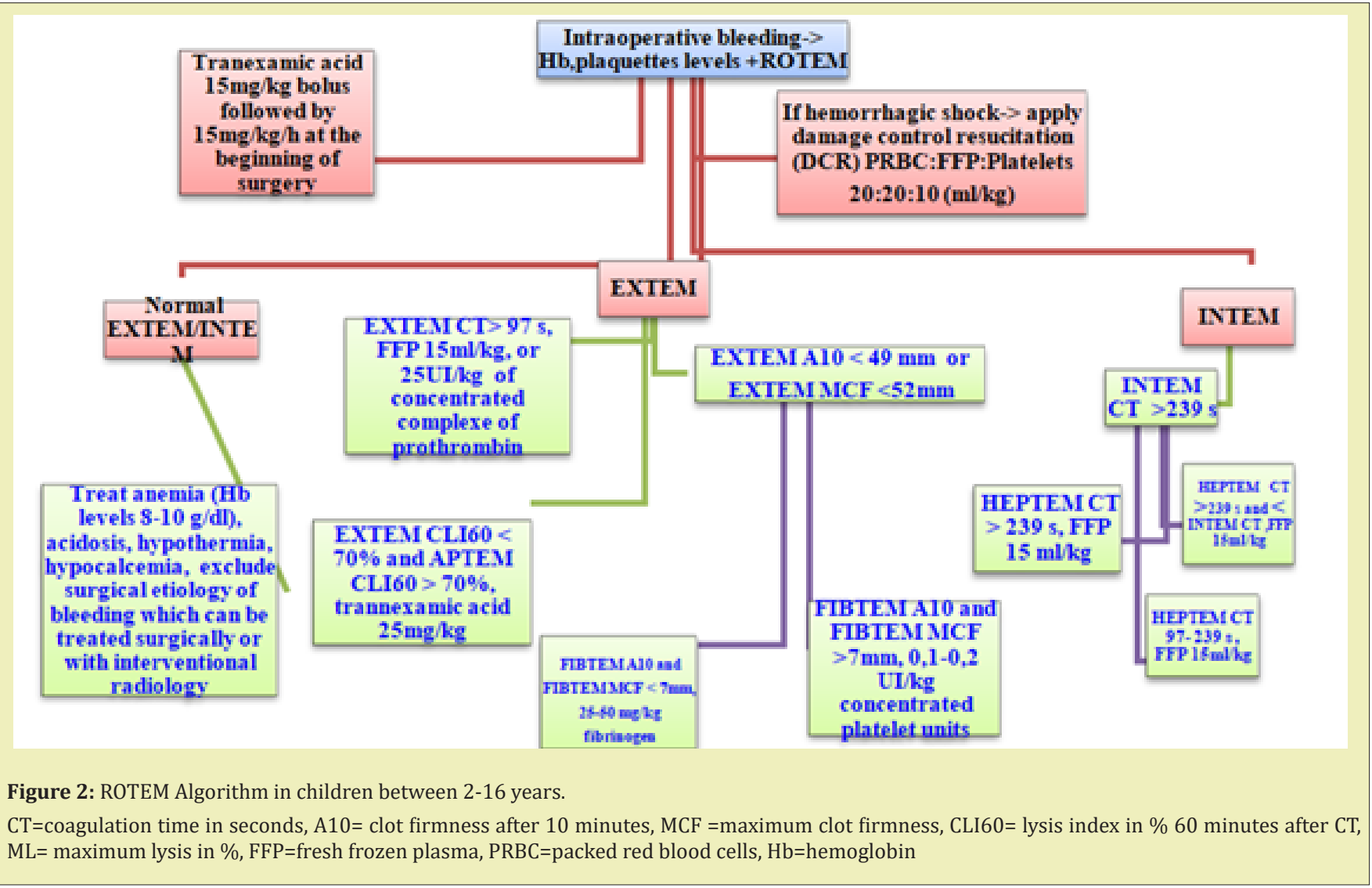




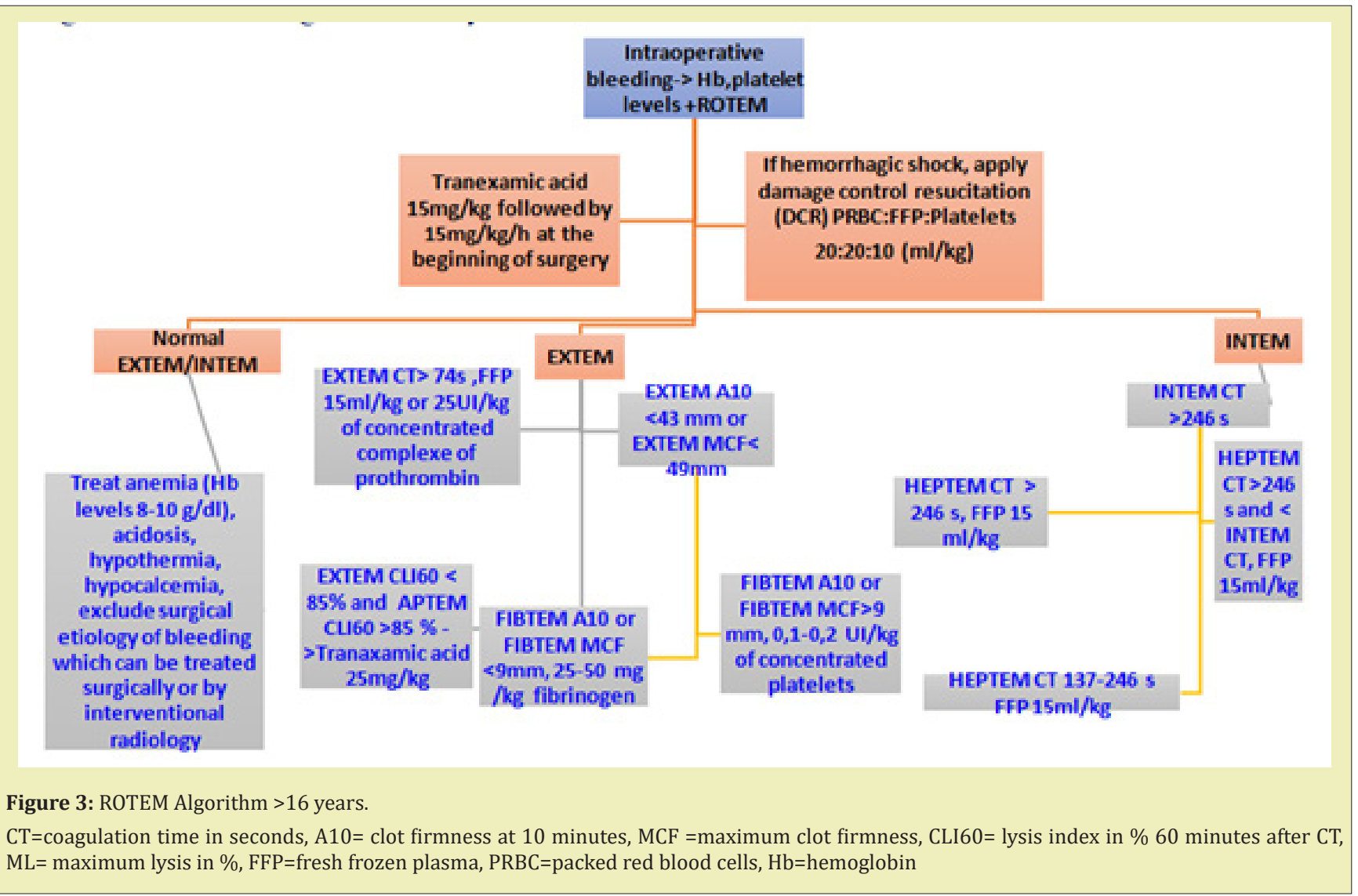

\section{Conclusion}

Liver transplantation is a major surgical setting with high intraoperative transfusion rates and postoperative complication rates in terms of organ dysfunction. There were multiple postoperative adverse outcome predictors. Optimal intraoperative patient management with transfusion guided by point-of-care viscoelastic devices and goal-directed fluid and hemodynamic therapy with validated parameters and tools in children is a possible pathway to upgrade postoperative evolution in pediatric liver transplantation.

\section{Ethics Approval}

This study received approval from the Ethics Committee of Necker on 21 March 2017 under registration number 2017-CK5-R1 and waived patient consent.

\section{Author's contributions}

Claudine Kumba conceptualized and designed the study and drafted the initial and final manuscript. She designed the data collection instruments, collected data, carried out initial and final analyses.

\section{Acknowledgements}

None.

\section{Funding}

None.

\section{Conflict of Interest}

The author declared no conflicts of interest.

\section{References}

1. Kumba C, Cresci F, Picard C, et al. Transfusion and Morbi-Mortality Factors: An Observational Descriptive Retrospective Pediatric Cohort Study. J Anesth Crit Care Open Access. 2017;8(4):00315.

2. Fanna M, Baptiste A, Capito C, et al. Preoperative risk for intra-operative bleeding in pediatric liver transplantation. Pediatr Transplantation. 2016;20:1065-1071.

3. Kumba C, Taright H, Terzi E, et al. Blood Product Transfusion and Postoperative Outcome in Pediatric Neurosurgical Patients. EC Anaesthesia. 2018;4(8):288-298.

4. Kumba C, Lenoire A, Cairet P, et al. Is Transfusion an Independent Risk Factor of Postoperative Outcome in Pediatric Orthopedic Surgical Patients ? A Retrospective Study. J Emerg Med Critical Care. 2018;4(2):7.

5. Kumba C, Querciagrossa S, Blanc Thomas, et al. Transfusion and Postoperative Outcome in Pediatric Abdominal Surgery. J Clin Res Anesthesiol. 2018;1(1):1-8.

6. Kumba C, Querciagrossa S, Harte C, et al. A Systematic Review and Meta-analysis of Goal Directed Intra-Operative Transfusion Protocols Guided by Viscoelastic Methods and Perioperative Outcomes in Children. Int J Recent Sci Res. 2019;10(03):31466-31471. 
7. Oswald E, Stalzer B, Heitz E, et al. Thromboelastometry (ROTEM) in children: age-related reference ranges and correlations with standard coagulation tests. British Journal of Anaesthesia. 2010;105(6):827835 .

8. Gan H, Cannesson M, Chandler JR, et al. Predicting fluid responsiveness in children: a systematic review. Anest Analg. 2013;117:1380-1392.

9. Pereira de Souza Neto E, Grousson S, Duflo F, et al. Predicting fluid responsiveness in mechanically ventilated children under general anaesthesia using dynamic parameters and transthoracic echocardiography. British Journal of Anaesthesia. 2011;106(6):856-864.

10. Biais M, Nouette Gaulain K, Roullet $S$, et al. A comparison os stroke volume variation measured by Vigileo/FloTrac System and Aortic Doppler Echocardiography. Anest Analg. 2009;109:466-469.

11. Slagt C, Malagon I, Groeneveld ABJ. Systematic review of uncalibrated arterial pressure waveform analysis to determine cardiac output and stroke volume variation. British Journal of Anaesthesia. 2014;112(4):626-637.

12. Bacchin MR, Ceria CM, Giannone S, et al. Goal-Directed Fluid Therapy Based on Stroke Volume Variation in Patients Undergoing Major Spine Surgery in Prone Position. Spine. 2016;41:E1131-E1137.

13. Kumba C, Willems A, Querciagrossa S, et al. A Systematic Review and
Meta- Analysis of Intraoperative Goal Directed Fluid and Haemodynamic Therapy in Children and Postoperative Outcome. J Emerg Med Critical Care. 2019;5(1):1-9.

14. Kumba C. Do Goal Directed Therapies Improve Postoperative Outcome in Children? (Perioperative Goal Directed Fluid and Hemodynamic Therapy; Transfusion goal directed therapy using viscoelastic methods and enhanced recovery after surgery and Postoperative outcome): A Study Research Protocol". Acta ScientificPaediatrics. 2019;2(7):17-19.

15. Kumba C. Physiology Principles Underlying Goal Directed Therapies in Children. Res Pediatr Neonatol. 2020;4(4). RPN.000591.

16. Kumba C. Rationale of Goal Directed Therapies in Children. Adv Pediatr Res. 2020;7:b42.

17. Kumba C. Trans-Thoracic Echocardiographic Aortic Blood Flow Peak Velocity Variation, Distance Minute, Aortic Velocity Time Integral and Postoperative Outcome in Pediatric Surgical Patients-An Observational Pilot Study Protocol. Open Journal of Internal Medicine. 2020;10:9095.

18. Kumba C. Goal directed fluid and hemodynamic therapy and postoperative outcomes in children: Value of transthoracic echocardiographic aortic blood flow peak velocity variation: A multi-centre randomized controlled trial protocol. Adv Pediatr Res. 2020;7:35. 